Ann. Sci. forest., 1970, 27 (2), 111-125.

\title{
RÉSULTATS APRÈS 9 ANS DE L'ESSAI DE FERTILISATION DE MOULIÈRE (VIENNE)
}

\author{
M. BONNEAU \\ avec la collaboration technique du personnel de l'Office National des Forêts \\ (Direction régionale de Poitiers) \\ Station de Recherches sur les Sols forestiers et la Fertilisation, \\ Centre national de Recherches forestières, 54 - Nancy \\ Institut national de la Recherche agronomique
}

RESUME

Une fertilisation minérale très abondante $\left(388 \mathrm{~kg}\right.$ de $\mathrm{P}_{2} \mathrm{O}_{5}, 160 \mathrm{~kg}$ de $\mathrm{K}_{2} \mathrm{O}$ et $800 \mathrm{~kg}$ de $\mathrm{CaO}$ par ha) a été pratiquée soit avant, soit quelques années après la plantation, sur pin Laricio de Corse (Pinus nigra Arn. ssp. Laricio Poiret). Le sol correspondant est un sol lessivé à pseudogley plus ou moins profond, argileux, très pauvre en phosphore assimilable et total. La végétation est essentiellement composée de Molinia coerulea et Calluna vulgaris.

Après 9 ans, le gain de croissance est de $50 \%$ en hauteur et $100 \%$ en circonférence. Ce gain semble correspondre sensiblement à un passage de la classe II à la classe I de fertilité. Malgré un coût initial élevé, que l'on pourrait sans doute réduire, cette fertilisation peut être rentable si le gain de fertilité se maintient pendant toute la durée de la révolution. Le taux de placement du capital investi en fertilisation serait de $4 \%$ environ.

La possibilité de financement des dépenses de fertilisation par accélération de la réalisation des vieux peuplements est discutée dans le cadre d'une futaie régulière à âges régulièrement gradués.

\section{1. - INTRODUCTION. RAPPEL DES RESULTATS ANTERIEURS}

La fertilisation minérale des forêts peut accrôtre leur production dans une proportion qui dépend du contexte écologique. Un inventaire expérimental des résultats possibles sur divers types de stations est nécessaire. L'essai de Moulière est un des premiers mis en place en France, bien qu'il ne date que de 1960. Il a déjà été rendu compte de ses premiers résultats (BonNEAU, 1963). Nous nous bornerons donc à rappeler brièvement les conditions d'installation et les premières réactions constatées. 


\section{1. - Description de la station}

L'expérience se situe à une vingtaine de kilomètres au nord-est de Poitiers, sur des alluvions anciennes argileuses de la Vienne, recouvertes d'une couche plus ou moins épaisse de limons. Les sols varient du pseudogley superficiel (bloc II) au sol lessivé à pseudogley (blocs I et III). Ils sont très pauvres en phosphore assimilable : 0,02 à $0,04 \%$ de $\mathrm{P}_{2} \mathrm{O}_{5}$ extrait par la méthode Duchaufour $\left(\mathrm{SO}_{4} \mathrm{H}_{2} \mathrm{~N} / 250\right.$, puis $\left.\mathrm{NaOH} \mathrm{N} / 10\right)$, ainsi qu'en phosphore semi-total : $0,10 \%$ de $\mathrm{P}_{2} \mathrm{O}_{5}$ extrait par $\mathrm{HCl}$ à $25 \%$ bouillant pendant 1 heure. La teneur en potassium échangeable est basse à moyenne : 0,08 à 0,13 m.e. pour $100 \mathrm{~g}$ de terre fine. Le $\mathrm{pH}$ est nettement acide et le rapport $\mathrm{C} / \mathrm{N}$ assez élevé en surface ( 22 à 26 en $\mathrm{A}_{0}, 18$ à 20 en $\mathrm{A}_{1}$ ). Le peuplement précédent était une futaie de pins sylvestre et maritime et la végétation, après la coupe, était surtout constituée de Molinia coerulea et Pteris aquilina. La callune s'est largement installée depuis dans les placeaux non fertilisés.

\section{2. - Traitements appliqués au début de l'essai}

L'essai comporte 3 blocs complets de 8 traitements; la variation entre blocs est importante. Les traitements appliqués peuvent se schématiser en 5 groupes.

\subsection{1. - Traitements 1 et 3.}

Aucune fertilisation. Dans 1, on a planté uniquement des pins Laricio de Corse (Pinus nigra Arn. ssp. Laricio Poiret) tandis que dans 3, on a introduit $1 / 6^{\mathrm{e}}$ de chêne rouge d'Amérique (Quercus borealis).

\subsection{2. - Traitements $2,4,8$.}

Fertilisation avec $92 \mathrm{~kg}$ d'azote (urée) par ha, épandus 1 mois avant plantation, $388 \mathrm{~kg}$ de $\mathrm{P}_{2} \mathrm{O}_{5}, 800 \mathrm{~kg}$ de $\mathrm{CaO}$ (scories et phosphates naturels) et $160 \mathrm{~kg}$ de $\mathrm{K}_{2} \mathrm{O}$ (chlorure de potassium), enfouis par labour au cours de l'automne qui a précédé la plantation. Les traitements 4 et 8 diffèrent du traitement 2 par introduction de $1 / 6^{\mathrm{e}}$ de chêne rouge. Dans le traitement 8 , le sol a subi un sous-solage avant labour.

\subsection{3. - Traitement 5 .}

Identique au traitement 4 , mais on a détruit la molinie par application de Dalapon l'année avant la plantation.

\subsection{4. - Traitement 6 .}

Il n'a pas été fertilisé au début mais a bénéficié du traitement herbicide.

\subsection{5. - Traitement 7 .}

Comme 4, mais on a cultivé et enfoui en 1959, l'année précédant la plantation, un engrais vert (lupin). 


\section{3. - Résultats à la fin de 1962}

La hauteur totale atteinte à la fin de 1962 faisait apparaître un effet très net de la fertilisation et du traitement herbicide. L'action du sous-solage paraissait nulle et celle de l'engrais vert faible.

\section{2. - TRAITEMENTS COMPLEMENTAIRES ET RESULTATS EN 1968}

\section{1. - Traitements complémentaires}

A partir de 1963 , on a complété le traitement 6 et splité tous les traitements pour tester l'effet d'un nouvel apport d'engrais azoté.

2.11. - Dans le traitement 6 , en 1963 , on a appliqué à chaque plant, alors au début de sa $4^{\mathrm{e}}$ année de végétation après plantation, $17 \mathrm{~g}$ de $\mathrm{P}_{2} \mathrm{O}_{5}$ et $17 \mathrm{~g}$ de $\mathrm{K}_{2} \mathrm{O}$, sous forme de phosphate naturel potassique. En 1964, la même dose a été appliquée sous forme de superphosphate potassique, puis $25,5 \mathrm{~g}$ en 1965 , sous la même forme. Enfin, en 1966, on a effectué un nouvel épandage de superphosphate sur toute la surface des placeaux. Le total de ces applications s'élève à $480 \mathrm{~kg}$ de $\mathrm{P}_{2} \mathrm{O}_{5}$ et $220 \mathrm{~kg}$ de $\mathrm{K}_{2} \mathrm{O}$ par ha, soit sensiblement plus que dans les autres traitements fertilisés (ceci par suite d'une erreur de calcul car le projet initial prévoyait des doses égales).

2.12. - Tous les placeaux ont été divisés en deux sous-placeaux, l'un (sous-traitement $\mathrm{O}$ ) ne recevant rien, l'autre (sous-traitement $\mathrm{N}$ ) recevant une fertilisation azotée appliquée sous forme d'ammonitrate à $34,5 \%$, d'abord par pied (4,6 g de $\mathrm{N}$ en 1963), puis sur toute la surface des sous-placeaux $(50 \mathrm{~kg}$ de $\mathrm{N}$ par ha en $1964,50 \mathrm{~kg}$ en 1965 , $50 \mathrm{~kg}$ en 1966).

\section{2. - Résultats}

Sans entrer dans le détail, disons que le traitement 6, qui s'était différencié du traitement 1 (effet de l'herbicide sans fertilisation) jusqu'en 1962, ne se différenciait plus de 1 ni de 3 en 1963, ce qui montrait que le désherbage chimique sans fertilisation n'avait pas d'effet durable dans cette station. L'accroissement en hauteur en 1963 était de $8,4 \mathrm{~cm}$ pour le traitement 1 , de $9,3 \mathrm{~cm}$ pour le traitement 6 et de $9,4 \mathrm{~cm}$ pour le traitement 3 , alors que la plus petite différence significative entre traitements était de $5,39 \mathrm{~cm}$.

Pour simplifier, nous ne donnerons et commenterons que les résultats à la fin de 1968, sur la hauteur totale, la circonférence à $1,30 \mathrm{~m}$ et l'accroissement de hauteur de 1966 à 1968.

\subsection{1. - Hauteur globale à la fin de 1968.}

Les résultats par bloc, traitement et sous-traitement sont donnés au tableau 1. 
TABLEAU 1

Hauteur à la fin de 1958 en mètres

TABLE 1

Heights at the end of 1968, m

Column (1): Mean height of the sub-treatments.

(2): Differences between sub-treatments $\mathrm{O}$ and $\mathrm{N}$.

(3): Mean height of the treatments.

\begin{tabular}{|c|c|c|c|c|c|c|c|}
\hline \multirow{2}{*}{$\begin{array}{l}\text { Traite- } \\
\text { ments }\end{array}$} & \multirow{2}{*}{$\begin{array}{l}\text { Sous- } \\
\text { traite- } \\
\text { ments }\end{array}$} & \multicolumn{3}{|c|}{ Blocs } & \multirow{2}{*}{$\begin{array}{l}\text { Moyenne } \\
\text { (1) }\end{array}$} & \multirow{2}{*}{$\begin{array}{l}\text { Différences } \\
\text { entre } \\
\text { sous- } \\
\text { traitements } \\
(\mathrm{N}-\mathrm{O})(2)\end{array}$} & \multirow{2}{*}{$\begin{array}{l}\text { Moyenne } \\
\text { des } \\
\text { traitements } \\
\text { (3) }\end{array}$} \\
\hline & & I & II & III & & & \\
\hline \multirow{2}{*}{$1 \ldots$} & $\mathrm{O}$ & 1,97 & 1,52 & 2,13 & 1,87 & \multirow{2}{*}{$-0,28$} & \multirow{2}{*}{1,73} \\
\hline & $\mathrm{N}$ & 1,66 & 1,40 & 1,71 & 1,59 & & \\
\hline \multirow{2}{*}{$2 \ldots$} & & 2,81 & 2,25 & 3,74 & 3,26 & \multirow{2}{*}{$+0,01$} & \multirow{2}{*}{3,27} \\
\hline & $\mathrm{N}$ & 3,90 & 2,31 & 3,62 & 3,27 & & \\
\hline \multirow{2}{*}{$3 \ldots$} & $\mathrm{O}$ & 2,85 & 1,31 & 2,46 & 2,20 & \multirow{2}{*}{$-0,23$} & \multirow{2}{*}{2,08} \\
\hline & $\mathrm{N}$ & 2,35 & 1,25 & 2,31 & 1,97 & & \\
\hline \multirow{2}{*}{$4 \ldots$} & $\mathrm{O}$ & 3,21 & 2,44 & 3,47 & 3,04 & \multirow{2}{*}{$+0,28$} & \multirow{2}{*}{3,18} \\
\hline & $\mathbf{N}$ & 3,78 & 2,79 & 3,40 & 3,32 & & \\
\hline \multirow{2}{*}{5} & $\mathrm{O}$ & 3,74 & 2,47 & 3,75 & 3,32 & \multirow{2}{*}{$+0,32$} & \multirow{2}{*}{3,48} \\
\hline & $\mathbf{N}$ & 3,82 & 3,00 & 4,12 & 3,64 & & \\
\hline \multirow{2}{*}{6} & $\mathrm{O}$ & 2,82 & 1,97 & 2,68 & 2,49 & \multirow{2}{*}{$+0,27$} & \multirow{2}{*}{2,62} \\
\hline & $\mathbf{N}$ & 3,14 & 2,16 & 3,00 & 2,76 & & \\
\hline \multirow{2}{*}{7} & $\mathrm{O}$ & 2,76 & 2,71 & 3,45 & 2,97 & \multirow{2}{*}{$+0,37$} & \multirow{2}{*}{3,15} \\
\hline & $\mathrm{N}$ & 3,27 & 2,85 & 3,91 & 3,34 & & \\
\hline \multirow{2}{*}{8} & $\mathrm{O}$ & 3,36 & 2,48 & 3,60 & 3,14 & \multirow{2}{*}{$+0,06$} & \multirow{2}{*}{3,17} \\
\hline & $\mathrm{N}$ & 3,28 & 2,57 & 3,76 & 3,20 & & \\
\hline
\end{tabular}

2.211. - Effet des traitements principaux. - Le tableau 2 indique les différences significatives entre les moyennes des traitements pour l'ensemble des deux sous-traitements $\mathrm{O}$ et $\mathrm{N}$. On voit clairement apparaître 3 groupes signifizativement différents les uns des autres :

- traitements sans fertilisation: 1 et 3 ;

--- traitement 6 fertilisé en surface seulement après la plantation ;

- traitements 2, 4, 5, 7, 8 fertilisés avant la plantation.

Aucun traitement ne diffère des autres à l'intérieur de ces groupes. La moyenne de l'ensemble des traitements fertilisés, sans herbicide et sans apport d'azote après plantation (2-0, 4-0, 7-0, 8-0), s'établit à 3,10 m contre 2,03 m pour l'ensemble des témoins (1-0 et 3-0), soit un gain de $52 \%$. 
TABLEAU 2

Hauteur totale fin 1968 : différences entre traitements. Les différences significatives à 1 \% sont soulignées 2 tois. Les différences significatives à $5 \%$ sont soulignées 1 fois

TABLE 2

Heights at the end of 1968: differences between treatments. Significant differences at 1 \% level: twice underlined. Significant differences at $5 \%$ level: once underlined

\begin{tabular}{|c|c|c|c|c|c|c|c|}
\hline & 3 & 6 & 7 & 8 & 4 & 2 & 5 \\
\hline 1 & 0,35 & 0,89 & 1,42 & 1,44 & 1,45 & 1,54 & 1,75 \\
\hline 3 & & 0,54 & 1,08 & 1,10 & 1,11 & 1,20 & 1,41 \\
\hline 6 & & & 0,53 & 0,55 & 0,56 & 0,65 & 0,86 \\
\hline 7 & & & & 0,02 & 0,03 & 0,12 & 0,33 \\
\hline 8 & & & & & 0,01 & 0,10 & 0,31 \\
\hline 4 & & & & & & 0,09 & 0,30 \\
\hline 2 & & & & & & & 0,21 \\
\hline
\end{tabular}

Il est intéressant de constater que le traitement 6 se différencie très bien des traitements 1 et 3 : la fertilisation, même appliquée en surface et plusieurs années après la plantation, est parfaitement efficace, tout en l'étant moins que lorsqu'elle est appliquée au départ et enfouie.

Si on interprète l'effet des traitements à l'intérieur d'un même sous-traitement, on s'aperçoit que l'application d'azote effectuée de 1963 à 1966 ne change pas l'ordre d'efficacité des traitements, mais tend à les différencier davantage : le traitement 5 (herbicide + fertilisation) devient significatif au seuil de $1 \%$ par rapport au traitement 8 . L'absence de fertilisation azotée a l'effet inverse: à l'intérieur du sous-traitement 0 le traitement 6 ne se différencie pas de 3 et ne diffère de 1 qu'à $5 \%$.

Enfin, les résultats mettent en évidence l'influence de la différence de sols entre les blocs. Les moyennes des hauteurs des témoins (1-0 et 3-0) sont respectivement de $2,30 \mathrm{~m}$ dans le bloc III, le meilleur, et de $1,42 \mathrm{~m}$ dans le bloc II où le sol est le plus hydromorphe et le plus superficiel. L'effet de la fertilisation est le moins accusé dans le meilleur sol: dans le bloc III, la moyenne des placeaux fertilisés (2-0, 4-0, $7-0,8-0)$ s'établit à $3,74 \mathrm{~m}$ (+62\% par rapport aux témoins), contre 2,47 m dans le bloc II ( $74 \%$ par rapport aux témoins). La fertilisation minérale, malgré une forte action, ne peut donc pas compenser le handicap dû aux mauvaises propriétés physiques. Notons aussi que les gains dûs à la fertilisation dans les blocs II et III sont supérieurs à ceux constatés dans le bloc I, et ceci, bien que le sol du bloc III soit le meilleur. Cela tient à une légère hétérogénéité du bloc I dans lequel le traitement 3 se trouve favorisé, d'où un gain de $38 \%$ seulement des traitements fertilisés par rapport à la moyenne $1+3$. Ainsi, en prenant comme gain en hauteur la moyenne des trois blocs, nous sous-estimons plutôt l'effet de la fertilisation, si bien que les hypo- 
thèses faites à la fin de l'article pour les calculs économiques ne sont probablement pas trop optimistes.

2.212. — Effet des sous-traitements (cf. tableau I). — L'effet de la fertilisation azotée est significatif à $5 \%$ seulement pour l'ensemble des traitements et l'interaction traitement $\mathrm{x}$ sous-traitement est fortement significative. Il était donc justifié d'interpréter le dispositif d'une part sur les traitements ayant reçu une fertilisation phosphopotassique (et du calcium) et, d'autre part, sur les témoins. On aboutit dans les deux cas à un effet très significatif de l'apport d'azote, positif lorsque l'on a effectué un apport de PKCa, négatif dans le cas contraire. Cet effet dépressif de l'azote employé seul a été constaté dans beaucoup d'autres essais de fertilisation sur jeunes plantations, et on peut le tenir pour très général.

\section{TABLEAU 3}

Circonférences à $1 \mathrm{~m} 30$, en $\mathrm{cm}$, à la fin 1968

TABLE 3

Breastheight girth $(\mathrm{cm})$ at the end of 1968

Column (1) Mean girth of the sub-treatments.

(2) Differences between sub-treatments $\mathrm{O}$ and $\mathrm{N}$.

(3) Mean girth of the treatment.

\begin{tabular}{|c|c|c|c|c|c|c|c|}
\hline \multirow{2}{*}{$\begin{array}{l}\text { Traite- } \\
\text { ments }\end{array}$} & \multirow{2}{*}{$\begin{array}{l}\text { Sous- } \\
\text { traite- } \\
\text { ments }\end{array}$} & \multicolumn{3}{|c|}{ Blocs } & \multirow{2}{*}{$\begin{array}{l}\text { Moyenne } \\
\text { (1) }\end{array}$} & \multirow{2}{*}{$\begin{array}{l}\text { Différences } \\
\text { entre } \\
\text { sous } \\
\text { traitements } \\
\text { (N-O) (2) }\end{array}$} & \multirow{2}{*}{$\begin{array}{c}\text { Moyenne } \\
\text { des } \\
\text { traitements } \\
\text { (3) }\end{array}$} \\
\hline & & I & II & III & & & \\
\hline & $\mathrm{O}$ & 7,11 & 4,73 & 8,13 & 6,65 & & \\
\hline & $\mathrm{N}$ & 4,52 & 3,80 & 5,88 & 4,73 & & \\
\hline \multirow{2}{*}{2} & $\mathrm{O}$ & 18,60 & 10,18 & 15,70 & 14,82 & \multirow{2}{*}{$+1,60$} & \multirow{2}{*}{15,62} \\
\hline & $\mathrm{N}$ & 19,28 & 10,62 & 19,38 & 16,42 & & \\
\hline \multirow{2}{*}{3} & $\mathrm{O}$ & 12,71 & 2,41 & 12,29 & 9,13 & \multirow{2}{*}{$-1,83$} & \multirow{2}{*}{8,21} \\
\hline & $\mathrm{N}$ & 9,53 & 2,20 & 10,17 & 7,30 & & \\
\hline \multirow{2}{*}{4} & $\mathrm{O}$ & 17,34 & 11,83 & 18,74 & 15,97 & \multirow{2}{*}{$+2,17$} & \multirow{2}{*}{17,05} \\
\hline & $\mathrm{N}$ & 20,61 & 14,50 & 19,32 & 18,14 & & \\
\hline \multirow{2}{*}{5} & $\mathrm{O}$ & 20,01 & 11,13 & 19,67 & 16,93 & \multirow{2}{*}{$+3,12$} & \multirow{2}{*}{18,49} \\
\hline & $\mathrm{N}$ & 21,24 & 15,61 & 23,30 & 20,05 & & \\
\hline \multirow{2}{*}{6} & $\mathrm{O}$ & 13,72 & 7,24 & 12,80 & 11,25 & \multirow{2}{*}{$+2,23$} & \multirow{2}{*}{12,36} \\
\hline & $\mathrm{N}$ & 15,97 & 9,07 & 15,41 & 13,48 & & \\
\hline \multirow{2}{*}{7} & $\mathrm{O}$ & 13,32 & 13,90 & 19,98 & 15,73 & \multirow{2}{*}{$+1,94$} & \multirow{2}{*}{16,70} \\
\hline & $\mathrm{N}$ & 16,36 & 14,05 & 22,60 & 17,67 & & \\
\hline \multirow{2}{*}{8} & $\mathrm{O}$ & 19,08 & 12,34 & 20,15 & 17,19 & \multirow{2}{*}{$-0,28$} & \multirow{2}{*}{17,05} \\
\hline & $\mathrm{N}$ & 18,40 & 11,96 & 20,37 & 16,91 & & \\
\hline
\end{tabular}


L'effet positif de la fertilisation azotée reste cependant assez faible en valeur absolue puisque les moyennes des traitements $2,4,5,6,7$ et 8 s'établissent à $3,25 \mathrm{~m}$ en présence d'azote, et 3,03 $\mathrm{m}$ dans le cas contraire, soit un gain de $7 \%$ seulement. Sur le plan pratique, il ne semble pas que la fertilisation azotée vaille la peine d'être pratiquée, étant donné son coût.

\subsection{2. - Circonférence à $1,30 \mathrm{~m}$ à la fin de 1968 .}

Le tableau 3 donne les résultats par traitement, sous-traitement et bloc, le tableau 4, les différences significatives entre moyennes des traitements pour l'ensemble des sous-traitements $\mathrm{O}$ et $\mathrm{N}$.

\section{TABLEAU 4}

Circonférence à $1 \mathrm{~m} 30 \mathrm{en} \mathrm{cm}$ à la fin de 1968

Différences entre traitements :

- Différence significative à $1 \%$ soulignée 2 fois.

-- Différence significative à $5 \%$ soulignée 1 fois.

\section{TABLE 4}

Breastheight girth, $\mathrm{cm}$, at the end of 1968

Differences between treatments:

- Significant differences at $1 \%$ level: twice inderlined.

- Significant differences at $5 \%$ level: once inderlined.

\begin{tabular}{|c|c|c|c|c|c|c|}
\hline & 3 & 6 & 2 & 7 & $4=8$ & 5 \\
\hline $1 \ldots$. & 2,52 & 6,67 & 9,93 & 11,01 & 11,36 & 12,80 \\
\hline $3 \ldots$ & & 4,15 & 7,41 & 8,49 & 8,84 & 10,28 \\
\hline $6 \ldots$ & & & 3,26 & 4,34 & 4,69 & 6,13 \\
\hline $2 \ldots \ldots$ & & & & 1,08 & 1,43 & 2,87 \\
\hline $7 \ldots$ & & & & & 0,35 & 1,79 \\
\hline $4=8$ & & & & & & 1,44 \\
\hline
\end{tabular}

Les résultats sont les mêmes que pour les hauteurs: les groupes de traitements $(1,3),(6),(2,4,8,7,5)$ se différencient nettement entre eux sans qu'il y ait de différence à l'intérieur d'aucun des groupes. Toutefois, si l'on considère les résultats des traitements à l'intérieur du sous-traitement $\mathrm{N}$, le traitement 5 se révèle un peu meilleur que les traitements 2 et 8 . L'effet du traitement herbicide tend donc à se prolonger en présence d'une fertilisation complète NPKCa.

L'effet de l'azote est encore significatif, mais dépressif, sur les traitements 1 et 3 qui n'ont reçu aucune autre fertilisation, et positif sur l'ensemble des traitements qui ont reçu $\mathrm{P}, \mathrm{K}$ et $\mathrm{Ca}$.

Il faut noter que l'effet de la fertilisation sur les circonférences est beaucoup plus important que sur les hauteurs : la moyenne des placeaux 2-0, 4-0, 7-0 et 8-0 s'établit 
à $15,92 \mathrm{~cm}$ contre $7,89 \mathrm{~cm}$ pour la moyenne des placeaux témoins $1-0$ et $3-0$, soit un gain de $102 \%$. Cela revient à dire que la fertilisation change la forme des arbres; c'est à la fois intéressant car le gain en volume est plus important que ne l'indique le gain en hauteur seul, et inquiétant car le défilement est plus fort, ce qui augmente les chutes en scierie. De toute façon, ce défaut de forme peut se corriger dans l'avenir lorsque l'élagage naturel interviendra.

\subsection{3. - Somme des accroissements en hauteur des années 1967 et 1968.}

Elle a été obtenue par différence entre les mesures de 1968 et celles de fin 1966. Nous ne donnons que les moyennes par traitement et sous-traitement (tableau 5). L'interprétation statistique conduit aux mêmes résultats que pour les hauteurs et circonférences à la fin de 1968. On retrouve les trois groupes distincts: non fertilisés $(1,3)$, fertilisé en surface, après plantation (6), fertilisés avant plantation $(2,4,5$, 7,8 ), avec une tendance en faveur de 5 (fertilisation + herbicide), lorsque l'on considère l'effet des traitements à l'intérieur du sous-traitement $\mathrm{N}$. On peut donc considérer que les résultats à la fin de l'année 1968 ne sont pas dus seulement à la conservation des gains antérieurs, mais que l'effet des traitements est encore actuel. Le gain des traitements 2-0, 4-0, 7-0, 8-0 par rapport à 1-0 et 3-0 s'établit à 54\% .

\section{TABLEAU 5}

Somme des accroissements en hauteur des années 1967 et 1968 en mètres

TABLE 5

Sum of the height increase of the years 1967 and 1968

Column (1) Mean increase of the sub-treatments.

Line (2) Differences between sub-treatments inside each treatment.

\begin{tabular}{|c|c|c|c|c|c|c|c|c|c|}
\hline \multirow{2}{*}{$\begin{array}{l}\text { Sous- } \\
\text { traitements }\end{array}$} & \multicolumn{8}{|c|}{ Traitements } & \multirow{2}{*}{$\begin{array}{l}\text { Moyen- } \\
\text { nes (1) }\end{array}$} \\
\hline & 1 & 2 & 3 & 4 & 5 & 6 & 7 & 8 & \\
\hline$O \ldots \ldots \ldots$ & 0,62 & 1,17 & 0,77 & 1,09 & 1,20 & 0,89 & 1,00 & 1,03 & 0,97 \\
\hline $\mathrm{N} \ldots \ldots \ldots$ & 0,48 & 1,20 & 0,67 & 1,18 & 1,33 & 1,09 & 1,14 & 1,17 & 1,03 \\
\hline Moyenne ... & 0,55 & 1,19 & 0,72 & 1,13 & 1,27 & 0,99 & 1,07 & 1,10 & \\
\hline $\begin{array}{l}\text { Différence } \\
\mathrm{N}-\mathrm{O}(2) \ldots .\end{array}$ & $-0,14$ & $+0,03$ & $-0,10$ & $+0,09$ & $+0,13$ & $+0,20$ & $+0,14$ & $+0,14$ & \\
\hline
\end{tabular}

\subsection{4. - Analyses foliaires (voir tableau 6).}

Elles font très bien ressortir l'insuffisance d'alimentation en $\mathrm{N}$ et $\mathrm{P}$ dans les placeaux témoins 3-0, alors qu'il ne semble pas y avoir de problème de nutrition en potassium. L'amélioration due à la fertilisation apparaît nettement en 1966 dans le placeau III 2-N qui a reçu une fertilisation complète NPKCa. Elle est moins nette en 1968 , soit que l'effet de la fertilisation tende à diminuer (ce que contredisent les 
TABLEAU 6

Analyses foliaires en \% de la matière sèche

TABLE 6

Foliar analysis in percent of dry matter

\begin{tabular}{|c|c|c|c|c|c|}
\hline Bloc et traitement & Année & $\mathrm{N}$ & $\mathrm{P}_{22} \mathrm{O}_{5}$ & K & $\frac{\mathrm{N}}{\mathrm{P}_{2} \mathrm{O}_{\pi}}$ \\
\hline II $3-\mathrm{O} \ldots \ldots \ldots \ldots$ & 1963 & 1,21 & 0,18 & 0,58 & 7,0 \\
\hline III 3-O & 1968 & 1,38 & 0,20 & 0,65 & 7,0 \\
\hline III $2 \mathrm{~N} \ldots \ldots \ldots$ & 1966 & 1,56 & 0,33 & - & 4,8 \\
\hline III $2 \mathrm{~N} \ldots \ldots \ldots$ & 1968 & 1,22 & 0,24 & 0,65 & 5,0 \\
\hline III $2-\mathrm{O} \ldots \ldots$ & 1966 & 1,19 & 0,37 & - & 3,2 \\
\hline
\end{tabular}

accroissements de 1966 à 1968), soit qu'on se trouve en présence d'une fluctuation passagère due à l'année humide. On peut constater que, à travers ces fluctuations annuelles, le rapport $\mathrm{N} / \mathrm{P}_{2} \mathrm{O}_{5}$ conserve une constance assez remarquable: il est optimal et voisin de 5 dans les placeaux 2-N, trop élevé et voisin de 7 dans les témoins, ce qui explique bien l'effet marqué du phosphore et l'effet dépressif de l'azote employé seul. Les placeaux 2-0 ont un rapport $\mathrm{N} / \mathrm{P}_{2} \mathrm{O}_{5}$ de 3,2 , ce qui est trop faible et correspond à l'action positive de l'azote dans les placeaux qui ont déjà reçu du phosphore.

\section{3. - DISCUSSION ET CONCLUSIONS}

Ainsi, une fertilisation complète NPKCa a conduit à des gains de croissance très importants : $52 \%$ sur la hauteur, $100 \%$ sur la circonférence. Cet essai mériterait évidemment d'être contrôlé par plusieurs autres, mais nous pensons cependant que ses résultats sont applicables à une large gamme de sols voisins de ceux sur lesquels nous avons expérimenté, c'est-à-dire tous les sols lessivés hydromorphes développés sur les argiles à silex et leur couverture dans le Poitou, l'Anjou et la Touraine. Nous avons eu l'occasion de rencontrer beaucoup de ces sols dans la Sarthe et dans la région de Chinon.

Dans l'effet de cette fertilisation, l'acide phosphorique, associé au calcium et au potassium, semble avoir le rôle le plus important. Sur le plan pratique, les résultats montrent que l'on pourrait se passer de la fertilisation azotée appliquée après la plantation puisque son effet, bien que significatif, est faible en valeur absolue. Nous n'excluons pas cependant que de nouvelles applications, lorsque le peuplement sera plus âgé, puissent être bénéfiques. On pourrait se passer également des traitements annexes (herbicide, sous-solage, culture préalable de lupin) dont l'effet est faible ou nul. 
Dans ces conditions, une fertilisation identique à celle pratiquée dans les traitements 2-0 et 4-0 (388 kg de $\mathrm{P}_{2} \mathrm{O}_{5}, 160 \mathrm{~kg}$ de $\mathrm{K}_{2} \mathrm{O}, 800 \mathrm{~kg}$ de $\mathrm{CaO}, 100 \mathrm{~kg}$ de N$)$ coûterait $830 \mathrm{~F} /$ ha environ. C'est un coût élevé et on peut s'interroger sur la rentabilité de l'opération.

Il n'est pratiquement pas possible de répondre en toute certitude à cette question, dès aujourd'hui, puisque la réponse dépend en définitive du gain de production ligneuse

TABLEAU 7

Valeurs en fin de révolution des éclaircies capitalisées au taux de $4 \%$ et de la récolte principale

TABLE 7

Values at the end of the rotation of the capitalized thinning woods and of the final cutting

Column 1 Volume of thinning or final cutting.

Column 2 Price of the $\mathrm{m}^{3}$.

Column 3 Total price.

Column 4 Number of years of capitalization.

Column 5 Multiplication factor of capitalization.

Column 6 Value at the end of the rotation.

Column 7 Total value at the end of the rotation.

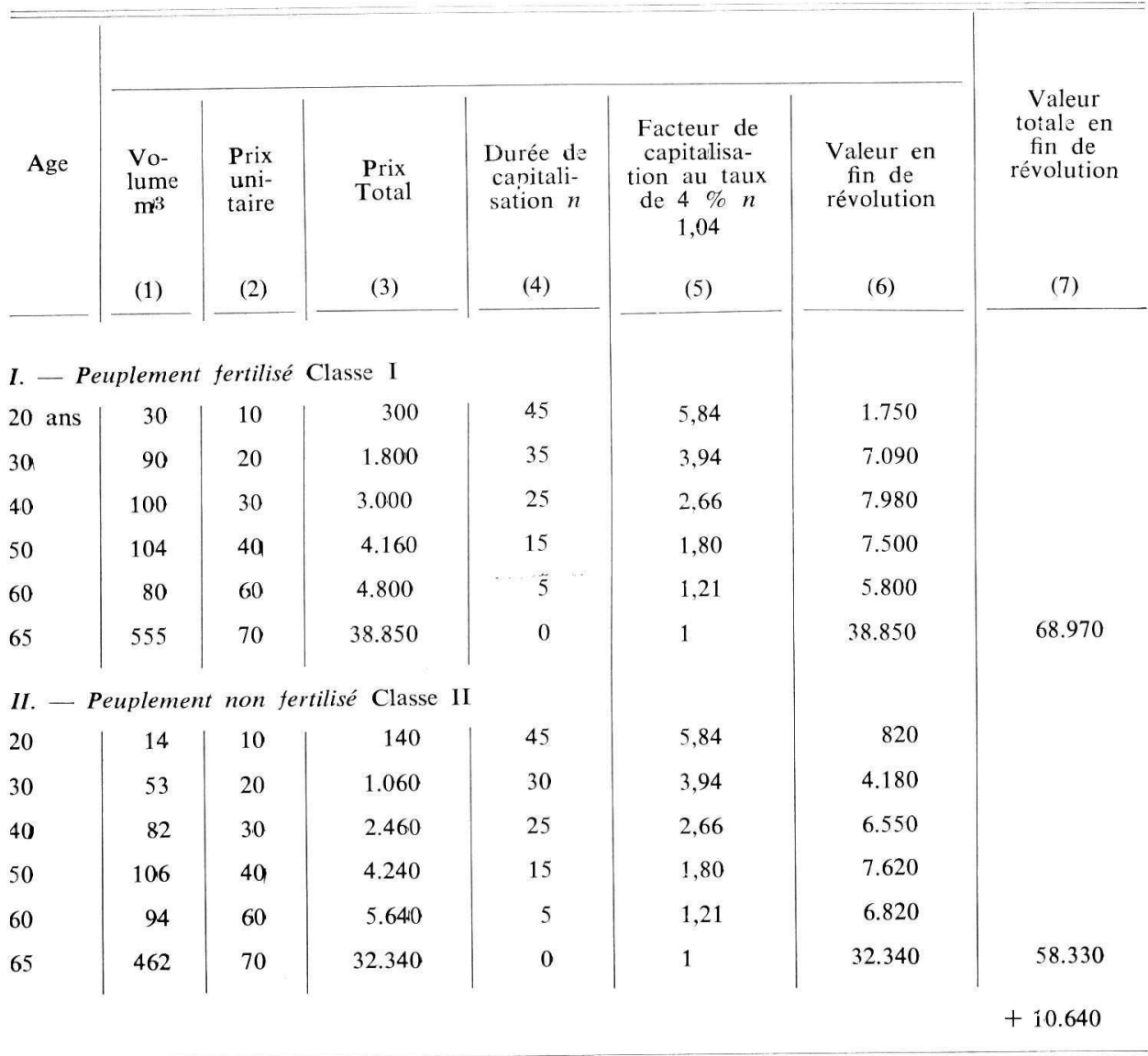


obtenu pendant toute la révolution. Nous avons cependant essayé de calculer la rentabilité en faisant l'hypothèse que le peuplement fertilisé gagne une classe de fertilité : il appartiendrait à la classe I de la table de production de N. Decourt, tandis que le témoin est de classe II. Nous admettons aussi que la fertilisation agira pendant toute la révolution (ce qui n'est évidemment pas prouvé pour le moment, mais n'est pas impossible étant donné les quantités très élevées d'engrais qui ont été apportées et la faiblesse des exportations comparées aux masses d'éléments mises en jeu dans le cycle biologique).

A partir de ces hypothèses, nous rechercherons si l'opération est rentable en envisageant deux cas.

\section{1. - Cas d'une parcelle isolée. Bilan cô̂ts-avantages actualisés}

Nous reprenons dans la table de Decourt les volumes coupés en éclaircie à 20 , $30,40,50,60$ ans pour le peuplement de classe I d'une part, et pour le peuplement de classe II (témoin) d'autre part, en leur appliquant des prix croissants avec l'âge du peuplement $\left(10 \mathrm{~F}\right.$ par $\mathrm{m}^{3}$ à 20 ans, $20 \mathrm{~F}$ à 30 ans, $30 \mathrm{~F}$ à 40 ans, $40 \mathrm{~F}$ à 50 ans, $60 \mathrm{~F}$ à 60 ans). Les valeurs des éclaircies sont capitalisées au taux de $4 \%$ jusqu'à la fin de la révolution, estimée à 65 ans (la table de production ne dépasse pas cet âge). Le volume restant sur pied à 65 ans est estimé à $70 \mathrm{~F}$ le $\mathrm{m}^{3}$ et son prix ajouté à celui des éclaircies capitalisées. Le tableau 7 donne le détail du calcul.

Nous arrivons à une valeur de $68970 \mathrm{~F}$ par ha pour le peuplement fertilisé et $58330 \mathrm{~F}$ pour le peuplement témoin. D'où un gain de $10640 \mathrm{~F}$. Si on le rapporte au coût initial de la fertilisation de $830 \mathrm{~F}$, supposé placé à intérêts composés pendant 65 ans, on aboutit à un taux de placement de $4 \%$, ce qui n'est pas très élevé, mais pas ridiculement bas en matière forestière.

La fertilisation pratiquée apparaît alors comme suffisamment rentable, malgré son coût initial élevé, si les hypothèses formulées sont exactes, ce que l'avenir seul pourra nous dire. On pourrait d'ailleurs appliquer des doses plus faibles à la plantation et les renouveler en cours de révolution. Le coût de la fertilisation en fin de révolution s'en trouverait réduit et les chances de constance du gain en volume probablement augmentées.

\section{2. - Cas d'une futaie régulière aménagée}

On a une suite de peuplements d’âge gradué. L'idée, développée par F. HoffmanN à propos de fertilisation de forêts adultes et appliquée ici au cas d'une plantation, consiste à récolter immédiatement le gain de production dû à la fertilisation, non pas sur les peuplements fertilisés eux-mêmes, mais sur les vieux peuplements de la forêt qui ne sont pas fertilisés, c'est-à-dire, finalement, à raccourcir la révolution.

Notre préoccupation est ici d'examiner comment on pourrait appliquer la fertilisation, de manière progressive, à l'ensemble de la forêt, au fur et à mesure des opérations de régénération artificielle (ou naturelle). Supposons que la révolution soit de 65 ans avant fertilisation; grâce à l'emploi des engrais, les peuplements appartenant désormais à la classe I auront à 60 ans le même volume sur pied que des peuplements non fertilisés de classe II à 66 ans. Nous pouvons donc, tout en conservant la même récolte en fin de révolution, raccourcir celle-ci de 66 à 60 ans. Les peuplements qui 
doivent être exploités entre l'année $n$, où la fertilisation est pratiquée sur la première parcelle, et l'année $n+60$, où cette parcelle passera en coupe définitive, devront donc être récoltés en 60 ans au lieu de 66 ans. Pendant ces 60 années, et ultérieurement, on exploite, au lieu de 1 ha dans l'ancien système, $1 \times \frac{66}{60}=1,1$ ha.

On peut calculer facilement que la récolte accélérée fournit dans ce cas un supplément de revenu très supérieur aux frais de fertilisation : $3465 \mathrm{~F}$ par an contre des frais de fertilisation de $913 \mathrm{~F}$, calculés sur 1,1 ha et non plus sur 1 ha. Mais ceci serait réalisé aux dépens du capital qui se trouve momentanément appauvri. On pourrait cependant accepter cette situation puisque le capital serait rétabli en fin de révolution (nous avons raccourci la révolution pour conserver ce capital égal à celui des anciens peuplements) et utiliser par exemple la différence entre les suppléments de récolte et les frais de fertilisation, à apporter des engrais sur des peuplements adultes quelques années avant leur coupe définitive, ce qui assurerait un nouveau profit.

On peut, plus raisonnablement, choisir un raccourcissement de révolution juste suffisant pour que le supplément de récolte couvre exactement les frais de fertilisation. On aboutit ainsi à un raccourcissement de révolution de 66 à 64 ans et à l'exploitation, chaque année, de 1,025 ha au lieu de 1 ha. Les peuplements de classe II ayant en fin de révolution $495 \mathrm{~m}^{3}$ sur pied, on récolte en plus chaque année $495 \times 0,025=12,4 \mathrm{~m}^{3}$ pour un prix de $12,4 \times 70=868 \mathrm{~F}$, couvrant un peu plus que les frais de fertilisation. En fait, quand nous arriverons à la réalisation des derniers peuplements non fertilisés, le supplément de récolte sera légèrement diminué, puisque les peuplements exploités auront 64 ans et non 66, et n'atteindra plus que $813 \mathrm{~F}$. Mais pour couvrir le reste des frais de fertilisation, nous pourrons compter alors sur un supplément d'éclaircie dans les peuplements déjà fertilisés.

On emprunte donc chaque année $868 \mathrm{~F}$ au capital de la forêt. En faisant l'hypothèse que $1,4 \mathrm{~m}^{3}$ du supplément de production ligneuse dû à la fertilisation reste sur pied chaque année, le reste étant enlevé en éclaircie, on peut calculer que, après 9 ans de fertilisation systématique des parcelles à régénérer, ce gain de production équilibre, en volume, la récolte supplémentaire : $9 \times 1,4 \times 1,025 \not 12,4 \mathrm{~m}^{3}$. Le volume sur pied de l'ensemble de la forêt passe donc par un minimum à ce moment, la diminution par rapport au capital antérieur n'étant que de $47 \mathrm{~m}^{3}: 9 \times 12,4-1,4 \times 1,025 \times$ $(1+2+\ldots \ldots+9)$ (nous avons 9 parcelles fertilisées ayant produit $1,4 \mathrm{~m}^{3}$ supplémentaire par an pendant $1,2, \ldots, 9$ ans).

En argent, la situation est la plus défavorable 26 ans après la fertilisation de la première parcelle, époque à laquelle la valeur d'avenir des suppléments de volume à obtenir en fin de révolution grâce à la fertilisation augmente exactement d'une valeur égale à l'emprunt fait annuellement au capital. En effet, la valeur d'avenir totale de ces suppléments, qui est la somme des valeurs d'avenir des suppléments à attendre d'une série de peuplements de $1,2, \ldots, 26$ ans, s'accroît chaque année du supplément de valeur d'avenir du peuplement le plus âgé. Le supplément de volume sur pied d'un peuplement de classe I à 64 ans, par rapport à un peuplement de classe II à 66 ans, est de $550-495=55 \mathrm{~m}^{3}$ et sa valeur d'avenir à 26 ans est :

$$
1,025 \times 55 \times 70 \times \frac{1}{1,04^{64-26}}=888 \mathrm{~F} \text {, }
$$

somme à peu près égale à l'emprunt fait au capital : $868 \mathrm{~F}$. 
Le total des suppléments de valeur d'avenir des 26 premiers peuplements fertilisés est alors :

$$
1,025 \times 70 \times 55 \times\left(\frac{1}{1,04^{38}}+\frac{1}{1,04^{39}}+\ldots \frac{1}{1,04^{64}}\right)=14600 \mathrm{~F} .
$$

Or, à ce moment, nous avons prélevé sur le capital une somme totale de $868 \times 26=$ $22568 \mathrm{~F}$, d'où une perte de capital de $8000 \mathrm{~F}$ environ, pour $66 \mathrm{ha}$, ce qui est très faible : $122 \mathrm{~F} /$ ha environ.

Après 64 ans de fertilisation, le capital sur pied se trouvera rétabli et même augmenté, puisqu'on n'aura plus dans la forêt, au lieu de peuplements de classe II, que des peuplements de classe I dont l'âge d'exploitabilité sera supérieur à 60 ans, âge auquel leur volume sur pied serait égal à celui de peuplements de classe II à 65 ans.

Autrement dit, en futaie régulière comportant des peuplements d'âge régulièrement gradués, on peut couvrir les frais de fertilisation par une accélération de la réalisation des vieux peuplements, opération qui ne comporte aucune baisse de revenu, mais se traduit seulement par une diminution temporaire et faible du capital sur pied. Ce capital, après une révolution complète, se trouve augmenté ainsi que les revenus puisqu'au cours de la révolution suivante nous couperons chaque année en coupe définitive, sans prélèvement sur le capital cette fois, 1,025 ha de peuplements de classe I à 64 ans au lieu de 1 ha de peuplement de classe II à 66 ans, soit $1,025 \times 540=550 \mathrm{~m}^{3}$ au lieu de $1 \times 495=495 \mathrm{~m}^{3}$, d'où une augmentation de revenu net (en déduisant les frais de fertilisation) de $55 \times 70-870=2980 \mathrm{~F}$. Encore ne tenons-nous pas compte d'une augmentation possible du prix $\mathrm{du}^{3}$ (diamètre plus gros) ni des récoltes en éclaircie qui sont également augmentées.

La baisse de capital momentanée constitue bien un petit risque si on est obligé de vendre la forêt avant reconstitution du capital sur pied, mais la perte maximale (112 F par ha) n'est pas même de l'ordre de grandeur d'une discussion normale de prix entre vendeur et acheteur. On peut même prétendre que cette baisse de capital n'existe pas si on prend le mot capital dans son sens de moyen de production générateur de revenus et non comme une réserve d'argent, puisque les revenus ne diminuent pas.

Nous pensons donc que la fertilisation des plantations, telle que nous l'avons pratiquée en forêt de Moulière, et malgré un prix élevé qu'on pourrait probablement réduire, est une opération intéressante et génératrice, à long terme, de profits. On doit considérer aussi que l'augmentation de matière première mise sur le marché ainsi d'ailleurs que la consommation d'engrais correspondante sont productrices, dans les secteurs secondaire et tertiaire de l'économie, de richesse et d'emploi et que la fertilisation est une parade efficace à la dégradation des sols sous plantation pure de résineux. Tout ceci bien entendu dans la mesure où l'avenir vérifiera les hypothèses techniques formulées.

Reçu pour publication en ịin 1970.

\section{SUMMARY}

RESULTS AFTER NINE YEARS OF THE ,FERTILIZING EXPERIMENT IN MOULIERE FOREST (VIENNE)

\section{1. - Description of the experiment}

The experiment is situated in the North of Poitiers, in West of France. The soil varies from a silty-clayey leached soil with deep pseudogley, to clayey shallow pseudogley. It is 
very deficient in phosphorus : 0,02 to $0,04 \%$ of assimilable $\mathrm{P}_{2} \mathrm{O}_{5}$ (extracted by $\mathrm{H}_{2} \mathrm{So}_{4}$ followed by $\mathrm{OH} \mathrm{Na} \mathrm{N} / 10$ ), and $0,10 \%$ of semi-total $\mathrm{P}_{2} \mathrm{O}_{5}$ extracted by hot $\mathrm{HCl} 25 \%$ during one hour. The previous stand was a forest of $P$. pinaster and $P$. silvestris with raw humus, which was clear-cut during winter 1958-59. The natural vegetation is mainly composed of Molinia caerulea and Calluna vulgaris.

The experiment has three complete blocs and eight treatments we can summarize as following :

\section{Treatments 1 and 3: no fertilizing. Control.}

Treatment 6 : Destruction of Molinia coerulea by Dalapon during the year before planting, in 1959. Spreading, from 4 th to 7 th year after planting of $480 \mathrm{~kg} / \mathrm{ka}$ of $\mathrm{P}_{2} \mathrm{O}_{5}, 220 \mathrm{~kg}$ of $\mathrm{K}_{2} \mathrm{O}$ as superphosphate and potassium chloride.

Treatments 2, 4, 5, 7, 8 : Ploughing the soil during the fall before planting and burying of $388 \mathrm{~kg} / \mathrm{ha}$ of $\mathrm{P}_{2} \mathrm{O}_{5}$ and $160 \mathrm{~kg} / \mathrm{ha}$ of $\mathrm{K}_{2} \mathrm{O}$ as basic slags, raw phosphate and potassium chloride, then $100 \mathrm{~kg} \mathrm{~N}$ as urea one month before planting.

Three years old pines (Pinus nigra Arn. Laricio Poiret were planted in spring 1960).

From 1963 all plots have been split in two sub-plots $O$ and $N$. The $O$ subtreatment received nothing; in the $\mathrm{N}$. subtreatment each tree received $15 \mathrm{~g}$ of ammonium-nitrate $(5,2 \mathrm{~g}$ of $\mathrm{N}$ ) in the spring 1963 ; then $50 \mathrm{~kg} / \mathrm{ha}$ of nitrogen, also in form of ammonium-nitrate, were broadcast in each spring 1964, 65, 66.

\section{2. - Results at the end of 1968}

2.1. Total height: Table 1 gives the means per bloc, treatment and subtreatment, table 2 gives the significant differences between treatments. The three groups of treatments $(1,3)$ not fertilized, (6) surface-fertilized after planting, $(2,4,5,7,8)$ with buried fertilizers before planting, differ at $1 \%$ level. Inside the same group, treatments do not differ from one another. The heights at the end of 1968 are the following :

$$
\begin{array}{ll}
1-0 \text { and } 3-0 & : 2,03 \mathrm{~m} \\
2-0,4-0,7-0,8-0 & : 3,10 \mathrm{~m} \text { that is a supplement of } 52 \% .
\end{array}
$$

The effect of nitrogen is negative on the treatments 1 and 3, which do not received P K Ca fertilizers, and positive on the treatment $2,4,5,6,7,8$ which received $\mathbf{P}$ K Ca either before or after planting, but the supplement is only $7 \%$.

2.2. Breast height girth (tables 3 and 4): The three groups of treatments differ significantly from one another as for the height. The supplement of growth due to the fertilizers is more important than for heights : $102 \%$. The mean girth is $7,89 \mathrm{~cm}$ for the treatments 1-0 and 3-0 and $15,92 \mathrm{~cm}$ for $2-0,4-0,7-0,8-0$. The effect of nitrogen is negative on the controls and positive on the fertilized treatments, but the supplement is not important.

2.3. Increase in height during the years 1967 and 1968 (table 5): The results are the same as for total height and prove the effect of the fertilizer is still present.

For practical use, fertilisation with $\mathrm{PK}$ and $\mathrm{Ca}$, given before planting, seems to be interesting, but one can do without nitrogen fertilizer given a few years after planting.

\section{3. - Profitability}

We suppose that the effect of fertilization will be active during the whole rotation, that not fertilized stands are of second class of fertility, and that the fertilized stands will be of first class.

3.1. First method of calculation: Using DECOURT's performance table, we calculate the capitalized value of thinning-wood at the end of rotation, with an annual rate of interest of $4 \%$. We add the value of final cutting. This calculation was made for a first class stand (fertilized) and for one of second class (not fertilized), the rotation being of 65 years. We obtain a difference of value of $10.640 \mathrm{~F}$. If fertilization costs are $830 \mathrm{~F}$ par ha, the annual rate of interest is about $4 \%$ (table 7).

3.2. - Second method: We think that the best growth of fertilized stands permits to shorten the rotation from 66 to 64 years. That permits to cut each year, as final cutting, 1,025 ha of old not fertilized stand instead of 1 ha, if the forest presents a good gradation of age classes. The supplement of crop is of $12,4 \mathrm{~m}^{3}$ every year and its value is $868 \mathrm{~F}$. So, we can have enough money to fertilize 1,025 ha of new plantation. 
This money is taken from the forest capital which undergoes an average impoverishment of $120 \mathrm{~F} / \mathrm{ha}$ after 26 years of practice of that new plan of cutting, but increases after that time. After the whole forest has been fertilized, that is to say after 64 years, the capital is greater than it was with the old not-fertilized stands. That momentary diminution of capital is very low. Moreover after the first cycle of fertilization, the net supplement of profit will reach $2.980 \mathrm{~F}$ every year (after deduction of fertilization costs of the parcel in course of regeneration).

\section{ZUSAMMENFASSUNG}

\section{ERGEBNISSE EINES NEUNJÄHRIGEN DÜNGUNGSVERSUCHS IN MOULIERE (VIENNE)}

Eine sehr starke mineralische Düngung (388 kg $\mathrm{P}_{2} \mathrm{O}_{5}, 160 \mathrm{~kg} \mathrm{~K}{ }_{2} \mathrm{O}$ und $800 \mathrm{~kg} \mathrm{CaO}$ pro ha) wurde entweder vor, oder eine Jahre nach der Pflanzung von Laricio-Kiefern (Pinus nigra Arn. ssp. Laricio Poiret) eingebracht. Der Boden ist ein mehr oder weniger tiefer, toniger Pseudogley und sehr arm an aufnehmbarem und totalem $\mathrm{P}_{2} \mathrm{O}_{5}$. Die Vegetation besteht vorwiegend aus Molinia coerulea und Calluna vulgaris.

Nach 9 Jahren ist ein Mehrzuwachs der Höhe von $52 \%$ und des Umfanges in Brusthöhe von $102 \% \mathrm{zu}$ verzeichnen. Diesen Mehrzuwachs scheint einem Uebergang der Bonität von der zweiten zur ersten Klasse entsprechen. Trotz ihrer höher Kosten, die man wahrscheinlich vermindern könnte, kann diese Düngung eine gute Rentabilität haben, wenn die Bonitätserhöhung während des ganzen Umtriebs andauert. Der Zinssatz des in der Düngung investierten Kapitals wäre ungefähr $4 \%$.

Die Möglichkeit der Finanzierung der Düngungskosten durch eine Beschleunigung des Einschlags der alten Bestände wird im Rahmen eines Hochwaldes mit guter Alterabstufung diskutiert.

\section{REFERENCES BIBLIOGRAPHIQUES}

Bonneau M., 1963. Premiers résultats d'une expérience de fertilisation sur Pin Laricio de Corse en forêt de Moulière. Ann. Ec. nat. Eaux et Forêts et de la St. Rech. Exp., XX, 3, 314-41.

Decourt N., 1965. Le Pin Sylvestre et le Pin Laricio de Corse en Sologne. Tables de production provisoires et méthodes utilisées pour les construire. Ann. Sci. forest., XXII, 2, 257-318.

Hoffmann F., 1969. Zur Frage der Rentabilität der Forstdüngung. Arch. Forstwes, 18, (7), 757-769. 